\title{
Solvation of atomic fluorine in bulk superfluid ${ }^{4} \mathrm{He}$
}

\author{
J. Eloranta \\ Department of Chemistry and Biochemistry, California State University, Northridge \\ 18111 Nordhoff St., Northridge, CA 91330, USA \\ E-mail: Jussi.Eloranta@csun.edu
}

Received December 1, 2010

\begin{abstract}
Bosonic density functional theory calculations were carried out for fluorine atom solvated in superfluid ${ }^{4} \mathrm{He}$ with an emphasis on the formation of dimeric species in the liquid. Atomic fluorine displays a relatively strong binding and anisotropic interaction with helium and hence the resulting solvation structure contains highly localized liquid helium layers. These solvent layers modify the gas phase dimer potentials by inclusion of a recombination barrier, which provides stabilization for the solvated fluorine atoms. At $0 \mathrm{~K}$ and saturated vapor pressure, the recombination barrier for the formation of molecular fluorine $\left({ }^{2} \Sigma_{g}^{+}\right)$in superfluid helium is predicted to be $26.8 \mathrm{~K}$. At temperatures below $1 \mathrm{~K}$, this barrier prevents the $\mathrm{F}-\mathrm{F}$ recombination as all the other electronic states correlating with the ground state atoms are essentially repulsive. It is concluded that it should be possible to stabilize fluorine atoms in superfluid helium below $1 \mathrm{~K}$ temperatures.
\end{abstract}

PACS: $36.40 . \mathrm{Mr}$ Spectroscopy and geometrical structure of clusters;

67.25.D- Superfluid phase.

Keywords: bulk superfluid helium, fluorine, quantum gel, solvation.

\section{Introduction}

Solvation of atomic and molecular impurities in bulk superfluid ${ }^{4} \mathrm{He}$ has been a subject to a number of experimental and theoretical studies [1-5]. On the experimental side absorption and fluorescence spectroscopy of solvated atoms have provided detailed information about the solvent structure surrounding the atomic impurities. Depending on the characteristics of the impurity-helium interaction, the resulting solvation structures can be classified according to two different ideal limits. The "bubble" structure is observed for impurities with mostly repulsive interaction with helium (e.g., alkali metal atoms) whereas the "snowball" structure forms around impurities exhibiting strongly bound potentials towards helium (e.g., ions). The strongly bound helium layer around the latter impurities often exhibits helium densities that approach the solid helium density. It was shown recently that such high-density solvent layers around atomic impurities may have important implications for the impurity recombination processes in the liquid [6]. As two impurities surrounded by high-density helium approach each other in the liquid, the gas phase interaction potential is altered mainly due to the repulsive interaction between the solvent layers on the two different centers. This effect was observed experimentally for the first time for doubly doped $\mathrm{Mg}$ containing helium droplets [7]. A theoretical investigation employing the bosonic den- sity functional theory later confirmed the interpretation of the experimental results [8]. In bulk superfluid helium experiments, it is possible to accumulate a large number of impurity centers in the sample, which may then lead to the formation of macroscopic size quantum gel-type structures [6]. It has been established that this type of structures are not related to the well-known impurity helium solids discovered by Gordon et al. $[6,9,10]$. Up to date no experimental observation of such quantum gel formation in the bulk has been published in the literature. To further elucidate the possible formation of quantum gel structures in the bulk, this study explores the solvation of fluorine atoms in bulk superfluid helium and provides estimates for the solvent layer induced energy barrier for F-F molecular recombination.

\section{Theory}

The applied density functional theory (DFT) to model bulk superfluid ${ }^{4} \mathrm{He}$ and the numerical implementation has been described previously [11-14]. The ground state solution was obtained by the imaginary time propagation method using variable time steps to speed up the convergence. The DFT model also included the high density corrections [12] to properly account for liquid localization in the bound parts of the fluorine-helium potential. For calculations at nonzero temperatures, the thermal DFT approach of Toigo et al. was used [15]. All calculations employed 
pair potentials based on the published ab initio data [1618]. Since the calculations were carried out in a finite 3D box, the number of helium atoms varied slightly depending on the positions of the fluorine atoms and the surrounding solvent layers. This was accounted for by normalizing the system to a constant number of helium atoms and then correcting the total energy appropriately. The zero-point spread for the solvated fluorine was included in the calculation by first optimizing its nuclear wavefunction alongside with the superfluid helium. The resulting fluorine atom density was nearly Gaussian with a full width at half height of 1.05 Bohr. In the subsequent calculations with multiple fluorine atoms their nuclear wavefunctions were kept fixed during the imaginary time propagation as the atomic centers are located far away from each other ensuring a minimal overlap between the nuclear wavefunctions.

\section{Results and discussion}

The formation of molecular fluorine in superfluid helium presents an interesting system because the atomic fluorine has a ${ }^{2} \mathrm{P}$ ground state and as such it exhibits an anisotropic interaction with the surrounding helium. Subsequently the dynamic Jahn-Teller effect should lead to a nonspherical solvation structure. It was recently discovered that the $\mathrm{F}_{2}{ }^{2} \Sigma_{g}^{+}$ground state potential has an unusual hump around $7.3 \mathrm{Bohr}$ with an approximate height of $12 \mathrm{~K}$ in the gas phase $[17,18]$. When this molecular recombination barrier is augmented with the solvent layer induced barrier, the effect becomes even more pronounced as demonstrated in Fig. 1. In superfluid helium $(T<2.17 \mathrm{~K})$, a barrier of $26.8 \mathrm{~K}$ is significant and should hinder thermally induced recombination of fluorine atoms towards $F_{2}$ $\left({ }^{2} \Sigma_{g}^{+}\right)$. Note that all the other states correlating with the ground state atoms are essentially repulsive and therefore only the ground state could lead to the formation of chemically bound $\mathrm{F}_{2}$.

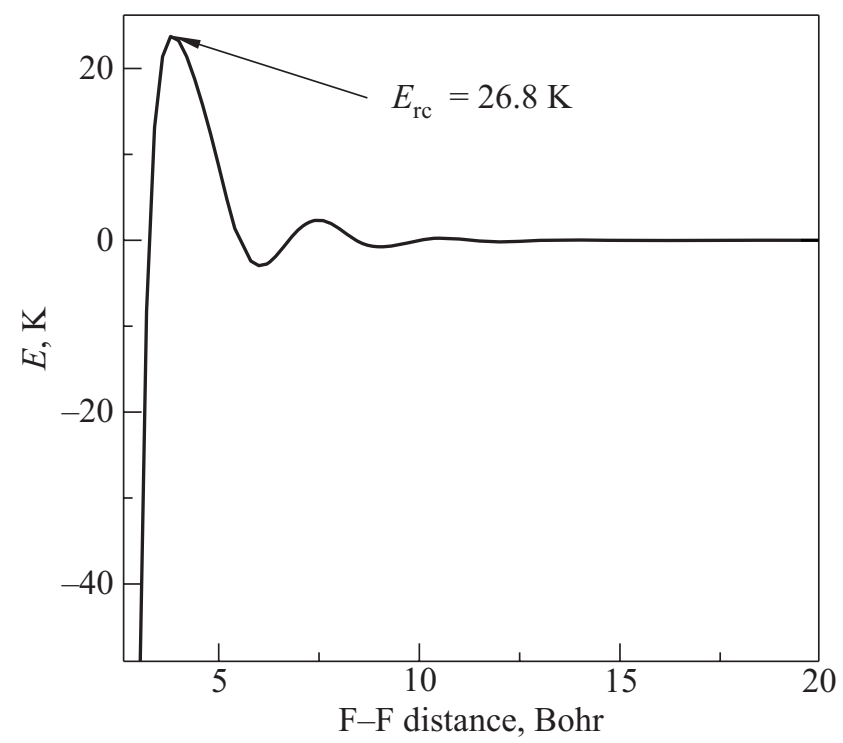

Fig. 1. F-F recombination potential in superfluid helium $\left({ }^{1} \Sigma_{g}^{+}\right)$.

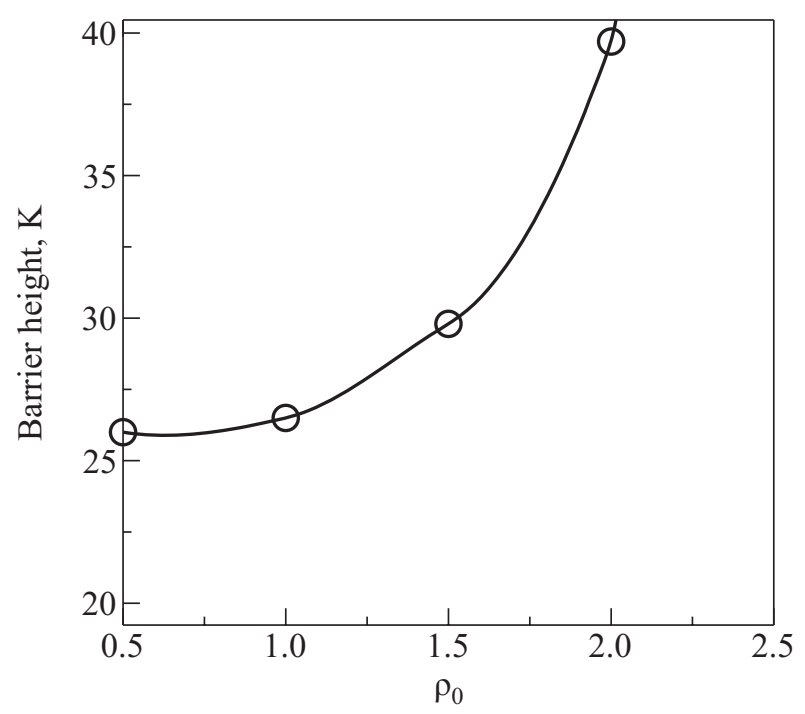

Fig. 2. F-F recombination potential barrier height as a function of bulk liquid density. $\rho_{0}$ represents the bulk liquid density at $0 \mathrm{~K}$ (see text).

By using the thermal DFT model, the effect of temperature on the solvent induced recombination barrier was calculated. However, since the liquid structure is dominated by the fluorine-helium pair potential, the thermal effects up to $3 \mathrm{~K}$ were found to be very small (less than $3 \mathrm{~K}$ ). On the other hand, the effect of increased pressure is more pronounced as the higher liquid density strongly amplifies the structure of the bound solvent layers and consequently the solvent layer induced recombination barrier becomes higher (see Fig. 2). At bulk liquid densities higher than $2.5 \rho_{0}$, where $\rho_{0}$ is the superfluid helium density at $0 \mathrm{~K}$ $\left(0.0218360 \AA^{-3}\right)$, a strongly inhomogeneous solid helium structure forms around the fluorine atoms. Due to the limited size of the simulation cube, it was not possible to study this region in detail at present. Ideally the atomic mobility would be greatly diminished in the limit of solid helium.

To understand the dynamics of thermal diffusion induced recombination of fluorine atoms in superfluid helium better, it is instructive to provide estimates for the second order rate constant for this process (i.e., $d[\mathrm{~F}] / d t=-k_{2}[\mathrm{~F}]^{2}$ where $[\mathrm{F}]$ is the fluorine atom concentration and $k_{2}$ is the 2nd order recombination rate constant). For reactive collisions, this can be estimated from $k_{2}=\sigma * \sqrt{8 k T / \pi \mu} N_{A} \exp \left(-E_{a} / R T\right)$ where $\sigma^{*}$ is the reactive cross-section (estimate $\approx 3 \cdot 10^{-2} \mathrm{~m}^{2}$ with a reactive diameter of $3 \AA$ and a steric factor of 1/9), $k$ is the Boltzmann constant, $\mu$ is the fluorine atomic mass, $N_{A}$ is the Avogadro's constant, $E_{a}$ is the solvent layer barrier height, and $R$ is the gas constant. Under saturated vapor pressure conditions, the resulting temperature dependency for $k_{2}$ is shown in Fig. 3. After the temperature exceeds the lambda point, the exponential term leads to a rapid increase in $k_{2}$ as temperature increases. The fluorine atom 


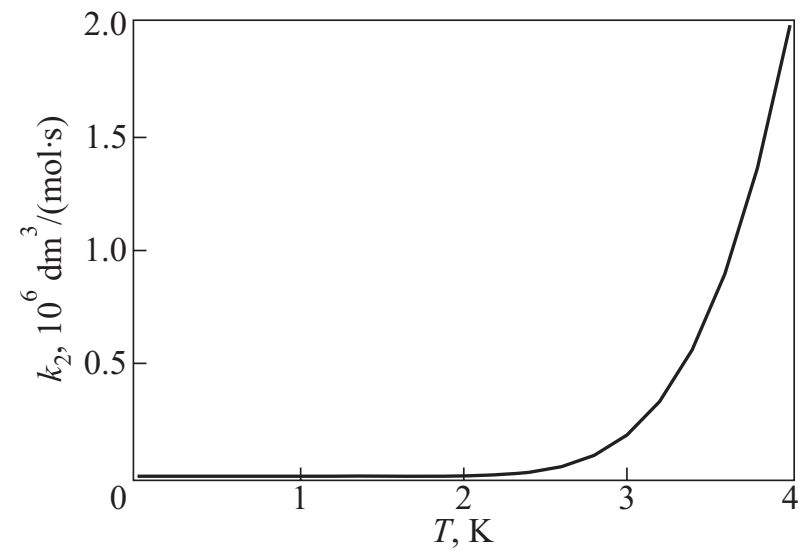

Fig. 3. Temperature dependence of the 2 nd order recombination rate constant $k_{2}$ predicted by collision theory.

concentration as a function of time at selected temperatures are plotted in Fig. 4. Below approximately $1 \mathrm{~K}$ temperature, the recombination kinetics is very slow allowing for fluorine atom buildup in bulk superfluid helium.

\section{Conclusions}

The present DFT calculations predict that the F-F molecular recombination barrier is sufficiently high so that isolated fluorine atoms can be stabilized in bulk superfluid helium below $1 \mathrm{~K}$ temperature.

Financial support from the National Science Foundation grant CHE-0949057 is gratefully acknowledged.

1. J.P. Toennies and A.F. Vilesov, Annu. Rev. Phys. Chem. 49, 1 (1998).

2. A. Hernando, R. Mayol, M. Pi, M. Barranco, F. Ancilotto, O. Bünermann, and F. Stienkemeier, J. Phys. Chem. A111, 7303 (2007).

3. Q. Hui and M. Takami, J. Low Temp. Phys. 119, 393 (2000).

4. T. Kinoshita, K. Fukuda, Y. Takahashi, and T. Yabuzaki, Phys. Rev. A52, 2707 (1995).

5. B. Tabbert, M. Beau, H. Günther, W. Häussler, C. Hönninger, K. Meyer, B. Plagemann, and G. zu Putlitz, Z. Phys. B97, 425 (1995).

6. J. Eloranta, Phys. Rev. B77, 134301 (2008).

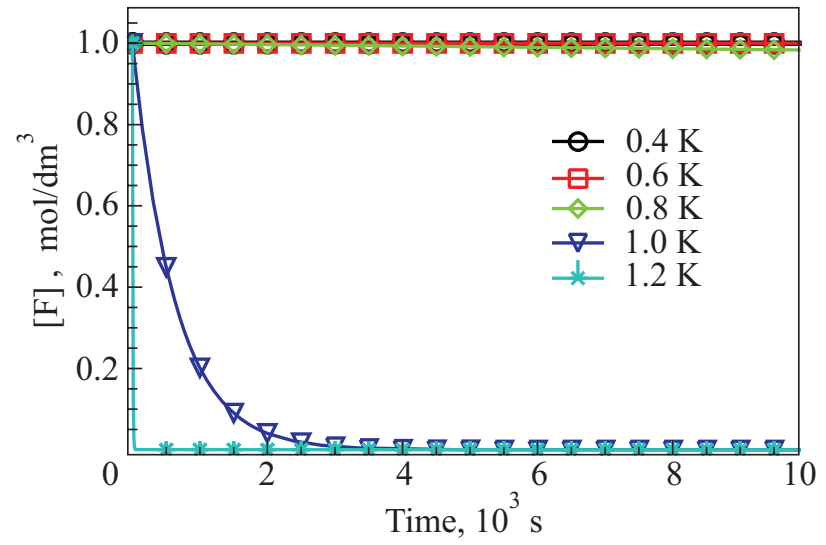

Fig. 4. Concentration of fluorine atoms $[\mathrm{F}]$ as a function of time at selected temperatures. The atoms are stabilized below $1 \mathrm{~K}$ temperature.

7. A. Przystawik, S. Göde, T. Döppner, J. Tiggesbäumker, and K.-H. Meiwes-Broer, Phys. Rev. A78, 021202 (2008).

8. A. Hernando, M. Barranco, R. Mayol, M. Pi, and F. Ancilotto, Phys. Rev. B78, 184515 (2008).

9. E.B. Gordon, L.P. Mezhov-Deglin, and O.F. Pugachev, JETP Lett. 19, 63 (1974).

10. E.B. Gordon and A.F. Shestakov, Fiz. Nizk. Temp. 26, 5 (2000) [Low Temp. Phys. 26, 1 (2000)].

11. F. Dalfovo, A. Lastri, L. Pricaupenko, S. Stringari, and J. Treiner, Phys. Rev. B52, 1193 (1995).

12. F. Ancilotto, M. Barranco, F. Gaupin, R. Mayol, and M. Pi, Phys. Rev. B72, 214522 (2005).

13. L. Lehtovaara, J. Toivanen, and J. Eloranta, J. Comp. Phys. 221, 148 (2007).

14. L. Lehtovaara, T. Kiljunen, and J. Eloranta, J. Comp. Phys. 194, 78 (2004).

15. F. Ancilotto, F. Faccin, and F. Toigo, Phys. Rev. B62, 17036 (2000).

16. H. Partridge, J.R. Stallcop, and E. Levin, J. Chem. Phys. 115, 6471 (2001).

17. L. Bytautas and K. Ruedenberg, J. Chem. Phys. 130, 204101 (2009).

18. F.A. Evangelista, E. Prochnow, J. Gauss, and H.F. Schaefer III, J. Chem. Phys. 132, 074107 (2010). 